\title{
Neuronal Subsets Express Multiple High-Molecular-Weight Cell-Surface Glycoconjugates Defined by Monoclonal Antibodies Cat-301 and VC1.1
}

\author{
Sam Zaremba, ${ }^{1}$ Janice R. Naegele, ${ }^{2}$ Colin J. Barnstable, ${ }^{1,2}$ and Susan Hockfield ${ }^{1}$ \\ Departments of 'Neuroanatomy and '2Ophthalmology and Visual Science, Yale University School of Medicine, New Haven, \\ Connecticut 06510
}

\begin{abstract}
Cat-301 and VC1.1 are monoclonal antibodies that recognize surface-associated molecules on subsets of mammalian CNS neurons. Earlier work demonstrated that Cat-301 recognizes a $680-\mathrm{kDa}$ chondroitin sulfate proteoglycan $(\mathrm{PG})$. VC1.1 has been shown to recognize 3 polypeptide bands on Western blot analysis; a major band at 95-105 kDa and additional bands at $145 \mathrm{kDa}$ and $170 \mathrm{kDa}$.

In the present report, we show that VC1.1 also reacts with a high-molecular-weight glycoconjugate. Immunoprecipitation experiments and biochemical characterizations indicate that Cat-301 and VC1.1 define at least 3 distinct high-molecular-weight antigens. The VC1.1 antigens react with antikeratan sulfate antibodies, while the Cat-301 antigens do not. By immunodepletion, we show that some VC1.1 antigens are Cat-301 positive, while others are Cat-301 negative. In addition, Cat-301-reactive proteoglycans are heterogeneous with respect to the presence or absence of VC1.1 epitopes. Double-label immunofluorescence studies with these 2 antibodies are consistent with the biochemical results and show that there are 3 classes of immunoreactive neurons in the cat CNS: Cat-301+/VC1.1 ${ }^{+}$, Cat-301-/VC1.1 and Cat-301+/2/C1.1 . These results indicate that structural microheterogeneity exists among Cat-301 and VC1.1 highmolecular-weight glycoconjugates. This heterogeneity may be a reflection of the diverse neuronal phenotypes that are recognized by Cat-301 and VC1.1 in the mammalian CNS.
\end{abstract}

The morphological diversity of neurons in the adult mammalian CNS has been recognized since the time of Ramon y Cajal. It has been suggested that this diversity is partially reflected by the selective expression of specific genes by different subpopulations of neurons. Such molecular diversity is likely to include surface macromolecules that might function in adhesive processes that subserve such phenomena as neuronal migration, axon fasciculation and guidance, target recognition, selective synapse formation, and synapse stabilization. In recent years, a

\footnotetext{
Received Jan. 23, 1990; revised Apr. 27, 1990; accepted May 1, 1990.

This work was supported by Grants EY06511 to S.H. and EY00785, EY05206, and EY07119 to C.J.B. S.H. and J.R.N. are Klingenstein Fellows in the Neurosciences. C.J.B. is a Jules and Doris Stein Research to Prevent Blindness Professor. The authors wish to thank Phillippe Malle, Richard LaPlante, and Bob Brown for photographic assistance and Hugh Fryer, Lawrence Molinaro, Jr., and Laura Milroy for technical help.

Correspondence should be addressed to Dr. Susan Hockfield, Section of Neuroanatomy, Yale University School of Medicine, 333 Cedar Street, New Haven, CT 06510 .

Copyright $(1990$ Society for Neuroscience $0270-6474 / 90 / 092985-11 \$ 03.00 / 0$
}

powerful approach for identifying cell-surface molecules on subpopulations of neurons has been the generation of specific immunological probes by hybridoma technology (Kohler and Milstein, 1975).

Cat-301 (Hockfield and McKay, 1983) and VCl.1 (Arimatsu et al., 1987) are 2 such monoclonal antibody probes that recognize antigens associated with the surface of mammalian CNS neurons. Each antibody outlines subsets of neurons throughout the neuraxis. Although the distributions of Cat-301 and VCl.1 immunoreactive neurons overlap in some areas, each antibody exhibits a distinctive pattern in other areas. For example, in the cat visual system, Cat-301 stains neuronal subsets in area 17 and the lateral geniculate nucleus (LGN), but not in the retina. In contrast, $\mathrm{VCl} .1$ selectively labels some neurons in area 17 and in the retina, but not in the LGN.

We and others have suggested that subset-specific surface markers such as Cat-301 and VC1.1 might recognize individual members of a biochemically distinct family of molecules. There is increasing evidence that high-molecular-weight (HMW) glycoconjugates on cell surfaces and in the extracellular matrix play critical roles in regulating cell-cell interactions during the development of the nervous system (for reviews, see, Ruoslahti, 1988; Margolis and Margolis, 1989). Previous studies have shown that the neuronal surface staining obtained with Cat-301 is due to the presence of a $680-\mathrm{kDa}$ chondroitin sulfate proteoglycan (Zaremba et al., 1989). In the present report, we show that VC1.1 also reacts with a similar HMW glycoconjugate. This HMW antigen shares some common structural features with the Cat301 protcoglycan, and, in fact, some of the HMW molecules react with both antibodies. However, several lines of evidence indicate that other HMW antigens react with one antibody but not the other. Double-label immunofluorescence confirms and extends these biochemical observations by showing that, in some CNS regions, VCl.1 and Cat-301 recognize different subsets of neurons, while in other regions, the 2 antibodies recognize the same neurons. These findings, along with other recently published reports (Watanabe et al., 1989; Fujita et al., 1989) are consistent with the hypothesis that distinct mammalian CNS neurons may express cell-type-specific surface HMW glycoconjugates, including proteoglycans.

\section{Materials and Methods}

Immunological reagents. The generation and subtyping of monoclonal antibodies Cat-301 (IgG) , Rat-302 (IgM) and VC1.1 (IgM) have been described previously (McKay and Hockfield, 1982; Arimatsu et al., 1987; Hockfield, 1987). Cat-301 and Rat-302 were used as an undiluted hybridoma supernatant, and VC1.1 was used as a diluted ascites fluid. 

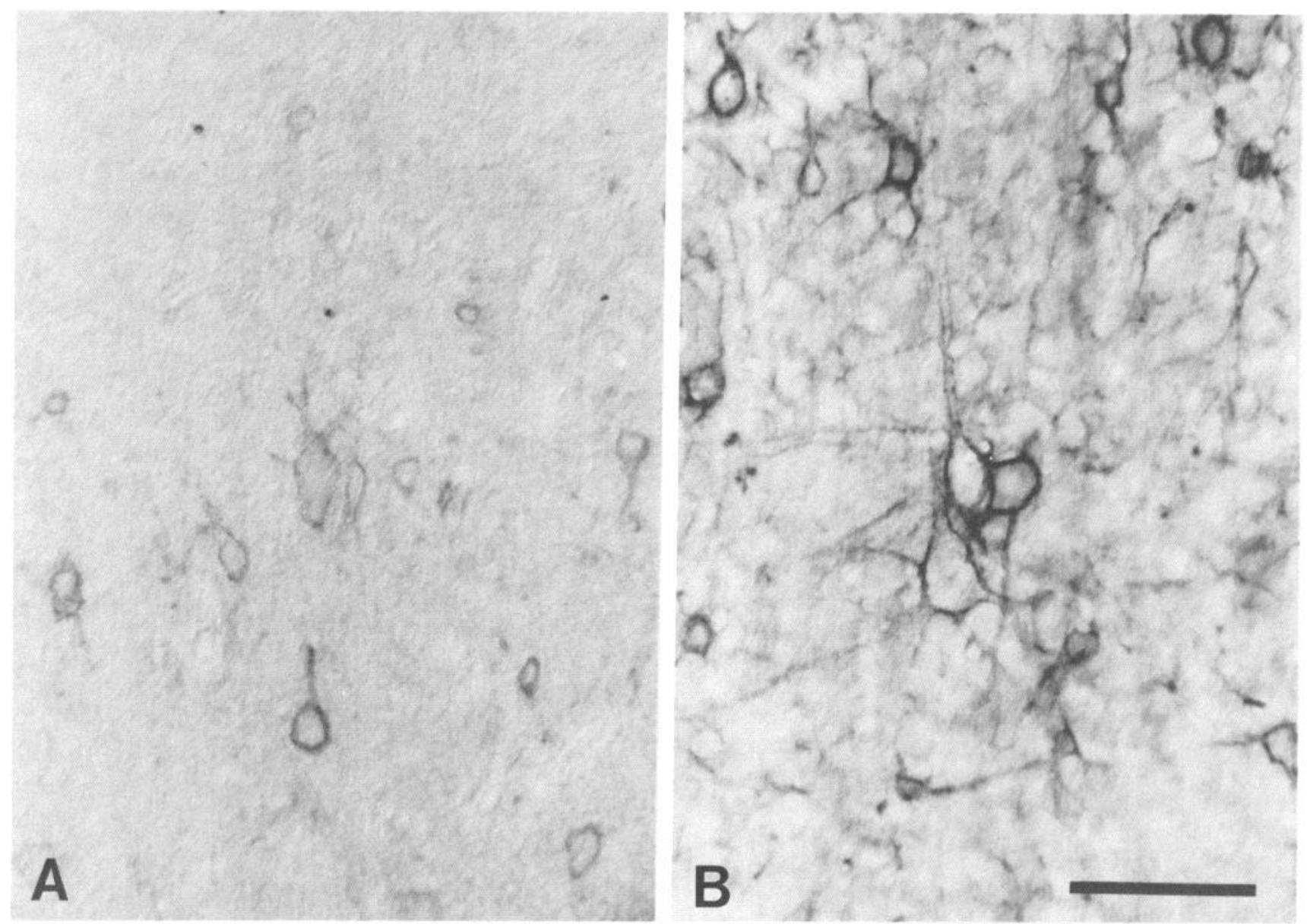

Figure 1. Immunoperoxidase localization of VC1.1 and Cat-301 on neuronal surfaces in cat visual cortex. A photomicrograph through layers 4 and 5 of the cat visual cortex showing that $\mathrm{VCl} .1(A)$ and Cat-301 $(B)$ recognized surface-associated or extracellular antigens on subsets of neurons. Scale bar, $100 \mu \mathrm{m}$.

Monoclonal antibody to keratan sulfate was obtained from ICN (Costa Mesa, CA). Previous cháracterizátiońs indicałte that this åntibody spécifically recognizes sulfated keratan sulfate moieties of a minimum hexasaccharide chain length (Caterson et al., 1983; Mehmet et al., 1986; Tang et al., 1986). The HNK-1 hybridoma cell line was obtained from American Type Culture Collection (Rockville, MD), and the antibody was used as a diluted ascites fluid. HNK-1 was subtyped as an IgM (Abo and Balch, 1981) and has been shown to recognize a carbohydrate antigen characterized as a sulfated glucuronic acid present on some glycolipids, as well as on several glycoproteins (Kruse et al., 1984; Chou et al., 1985).

Biochemical studies. All biochemical experiments were performed on fresh CNS tissue from adult cats. Preparation of homogenates, partial purification of antigens on DEAE and Sepharose CL-2B chromatography, and enzymatic digestions have been described previously (Zaremba et al., 1989).

For SDS-PAGE and Western blot analysis, some samples were concentrated by trichloroacetic acid (TCA) precipitation (Bensadoun and Weinstein, 1976). Samples were prepared for electrophoresis by boiling in $10 \mathrm{~mm}$ sodium phosphate (pH, 6.8), 3\% SDS, $10 \%$ glycerol, $0.01 \%$ bromophenol blue, and $1 \% \beta$-mercaptoethanol (SDS-PAGE buffer). Electrophoresis was performed according to the method of Laemmli (1970), using gradients of 3-8\% acrylamide in the resolving gels and $3 \%$ in the stacking gels. Gels were transferred to nitrocellulose (Towbin et al., 1979), with the modifications previously described (Zaremba et al., 1989). In the blotting experiments, the primary antibodies were used at 1:20,000-50,000 (VC1.1), 1:10,000 (anti-HNK-1), 1:500 (anti-keratan sulfate), and undiluted hybridoma supernatants (Cat-301 and Rat302 ). Binding of primary antibodies was detected using alkaline-phosphatase-conjugated secondary antibodies (anti-mouse IgG from Promega, anti-mouse IgM from Cappel).
Immunoadsorptions. Samples were dialyzed against $50 \mathrm{~mm}$ sodium phosphate $(\mathrm{pH}, 7.4)$ containing $0.15 \mathrm{M} \mathrm{NaCl}$ and $0.1 \% 3-[(3$-cholamidopropyl)dimethylammonio]1-propanesulfonate (CHAPS) to remove urea. Dialyzed samples were mixed overnight in the cold room with beads that had been precoated with the appropriate antibody. Cat-301 was adsorbed onto Protein G beads (Pharmacia) and VC1.1 (1:500 dilution) was adsorbed onto anti-mouse IgM Actigel (Sterogene, San Gabriel, CA). In the immunodepletion experiments, the unbound fraction from each round of adsorption was transferred to a fresh aliquot of the antibody-coated beads. Bound antigen was released from the beads for electrophoresis by boiling in SDS-PAGE buffer.

Immunofluorescence double labeling. Three adult cats were deeply anesthetized with pentobarbital and intracardially perfused with $0.1 \mathrm{M}$ sodium phosphate buffer ( $\mathrm{pH}, 7.4)$ containing $0.9 \%$ saline (PBS), followed by $4 \%$ paraformaldehyde in PBS. The brains were removed, blocked, and fixed an additional 1-5 hr by immersion in the same fixative. The blocks were then rinsed in PBS and cryoprotected according to the method of Eldred et al. (1983), immersed in Tissuetek (O.C.T. compound, Miles), frozen on dry ice, and stored at $-80^{\circ} \mathrm{C}$. Immunoperoxidase staining for Cat-301 or VC1.1 was done on $50-\mu \mathrm{m}$-thick, free-floating vibratome sections, using the avidin-biotin system (Vector Labs) as described previously (Naegele et al., 1988).

For double-label immunofluorescence staining, cryostat sections were cut at $12-15 \mu \mathrm{m}$ and dried onto gelatin-coated glass slides. Double labeling with VC1.1 and Cat-301 was carried out as described previously (Arimatsu et al., 1987), with slight modifications. The initial blocking step was performed with 5\% normal goat serum (NGS) in PBS for 30 min, then sections were incubated in a mixture containing Cat-301 hybridoma supernatant (diluted 1:10-40) and $\mathrm{VCl} .1$ ascites (diluted $1: 500-1000$ ) for $1 \mathrm{hr}$ at room temperature or overnight at $4^{\circ} \mathrm{C}$. These dilutions were determined optimal for obtaining strong staining with 


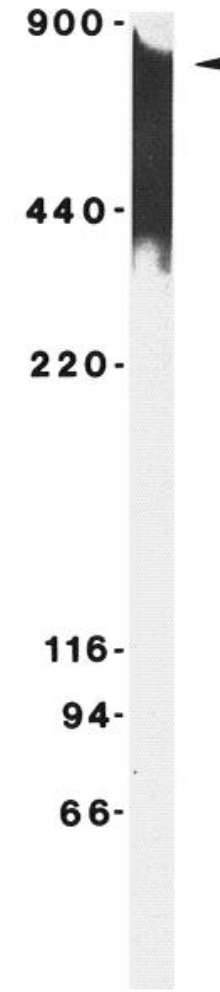

Cat 301

\section{VC1.1}

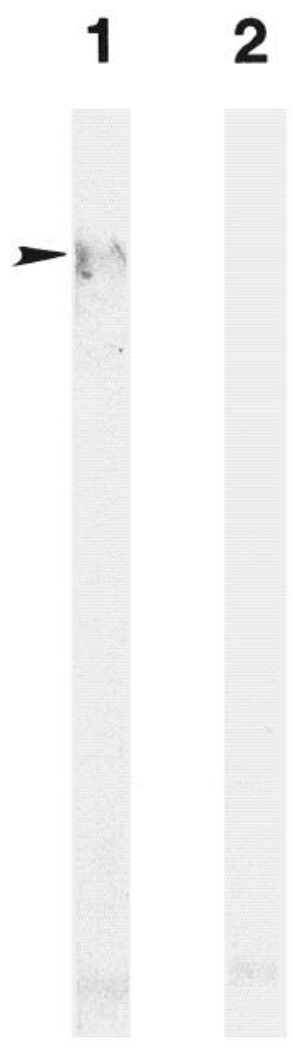

\section{C $x \quad$ Ret \\ Cat 301}

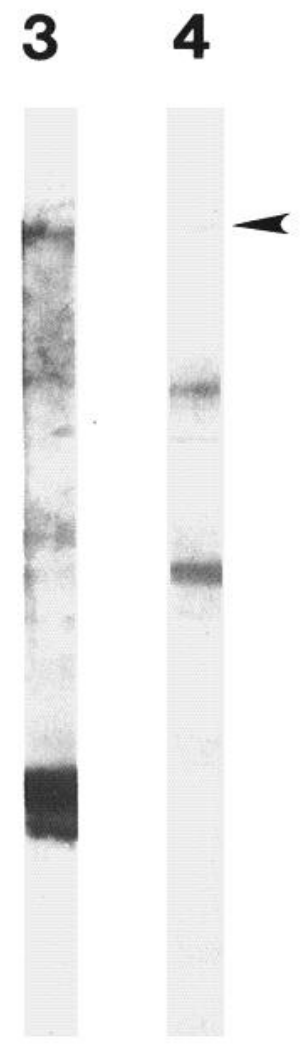

Cx Ret

\section{C 1.1} lecular weight standards were unreduced laminin $(900 \mathrm{kDa})$, laminin A and $\mathrm{B}$ subunits ( 440 and $220 \mathrm{kDa}$, respectively), $\beta$-galactosidase (116 $\mathrm{kDa})$, phosphorylase $\mathrm{B}(94 \mathrm{kDa})$, and bovine serum albumin $(68 \mathrm{kDa})$. Arrowheads indicate the positions of the Cat-301 and VC1.1 antigens.

both antibodies by comparing a wider range of dilutions in pilot studies. After incubation in primary antibody, sections were then washed in $5 \%$ NGS in PBS, incubated for $1 \mathrm{hr}$ at room temperature in a mixture of fluorescein isothiocyanate (FITC)-conjugated goat anti-mouse IgM ( $\mu$ chain specific, Sigma) and rhodamine isothiocyanate (RITC)-conjugated goat anti-mouse IgG Fc (Sigma). After extensive washing in PBS, sections were coverslipped in PBS and glycerol (1:1) and viewed using filters for FITC or RITC. As a control for specificity of the secondary antibodies, sections were incubated in monoclonal antibody VC1.1 or Cat-301 alone and then incubated with fluorescent secondary antibody mixture as described above. No cross-reactivity was found, and no significant background fluorescence was encountered. Stained sections were photographed with T-Max professional film (ASA 400) or Ecktachrome color slide film (ASA 600-1600).

To determine the proportions of neurons in cat area 17 that exhibited one or both of the VC1.1 and Cat-301 epitopes, 3 representative cryostat sections from area 17 of one animal were stained as described above, and the labeled neurons were scored for single or double immunofluorescence. The percentages of neurons in each category (Cat-301 ${ }^{+} / \mathrm{VCl} .1^{+}$, Cat $301^{-} / \mathrm{VCl}^{+} 1^{+}$, Cat-301+/VC1.1-) were then calculated.

\section{Results}

Cat-301 and VC1.1 were 2 of the first monoclonal antibodies reported to recognize surface-associated antigens on subsets of neurons in the mammalian CNS (Hockfield and McKay, 1983; Arimatsu et al., 1987). Staining with these 2 antibodies in the cat visual cortex is illustrated in Figure 1. Differences in the

Figure 3. Distribution of Cat-301 and VC1.1 antigens in cat cortex and retina. Homogenates of cat cortex (lanes 1,$3 ; 12.5 \mu \mathrm{g}$ total protein) or retina (lanes 2,$4 ; 57 \mu \mathrm{g}$ total protein) were electrophoresed, transferred, and blotted with Cat-301 (lanes 1,2) or VC1.1 (lanes 3, 4). Arrowheads indicate the positions of the $680-\mathrm{kDa}$ Cat-301 proteoglycan and the HMW VC1.1 antigen. Note the absence of the 95-105-kDa VC1.1 antigen in the retina, a tissue that shows intense neuronal staining with VC1.1

laminar distribution and in the numbers of immunoreactive neurons (see below) indicated that the distributions of Cat-301 and VC1.1 epitopes were only partially overlapping. Despite these differences, both antibodies gave remarkably similar staining when viewed at higher magnification. Each antibody produced a latticework pattern of staining that extended across the surface of neuronal cell bodies and onto proximal dendrites. At the electron microscopic level, Cat-301 and VC1.1 immunoreactivity was present extracellularly along intersynaptic regions of the plasma membrane and in the extracellular space surrounding synaptic terminal boutons, but was absent from synaptic clefts (see Figs. 2, 3 in Hockfield and McKay, 1983; Figs. 2, 3 in Naegele et al., 1988).

\section{Cat-301 and VC1.1 identify HMW antigens}

The similarity in surface staining patterns produced with these 2 antibodies prompted us to ask whether the respective antigens were similar biochemically. Aliquots of unfractionated cat cortex homogenates were electrophoresed and transferred under conditions that permitted immunodetection of HMW species. 


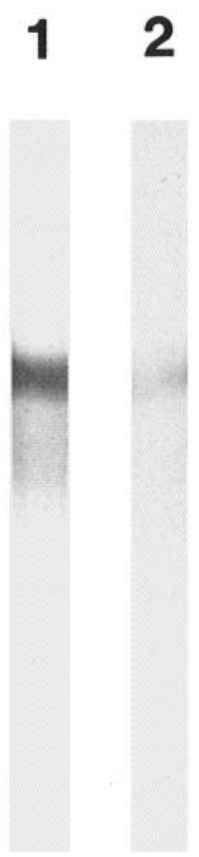

C V

Cat-301

\section{immunoprecipitate}

Figure 4. Cross-reactivity of immunoprecipitates with Cat-301 and VC1.1. Antigen partially purified through DEAE and Separose CL-2B (Zaremba et al., 1989) was immunoadsorbed with Cat-301-coated beads (lanes 1, 2) or VC1.1-coated beads (lanes 3, 4). Beads were washed, eluted, electrophoresed, transferred, and blotted with Cat-301 (lanes 1 , 4) or VCl.1 (lanes 2, 3). Each immunoprecipitate contained HMW antigens that reacted with both antibodies, but the Cat-301:VC1.1 staining ratio depended on the precipitating antibody.

Previous studies have shown that Cat-301 recognizes a 680 $\mathrm{kDa}$ chondroitin sulfate proteoglycan (Zaremba et al., 1989). VCl.1 was previously reported to recognize proteins at 95-105 $\mathrm{kDa}, 145 \mathrm{kDa}$, and $170 \mathrm{kDa}$ (Arimatsu et al., 1987). Figure 2 shows that VC1.1 (lane 2) also recognized an HMW antigen in addition to the previously described antigens. The size of the HMW VC1.1 antigen, as extrapolated from a calibration curve that included unreduced and reduced laminin, was approximately $650-700 \mathrm{kDa}$, a value close to that of the Cat-301 antigen. This VC1.1 species was not an artifact of nonspecific antibody binding, because the antigen was not detected with a control mouse IgM, Rat-302 (Fig. 2, lane 3). Through the remainder of this paper, the term "HMW VC1.1 antigen" will refer to this 650-700-kDa band.

In order to determine whether the HMW VC1.1 antigen might correspond to histologically detected antigens, Western blot analysis was carried out on homogenates from different brain regions (Fig. 3). The HMW VC1.1 band was detected in homogenates of the cortex and retina. In addition, VC1.1 also stained bands at $400 \mathrm{kDa}$ and $200 \mathrm{kDa}$, which were most prominent in tissue homogenates from the retina. The major band

\section{Cat-301}

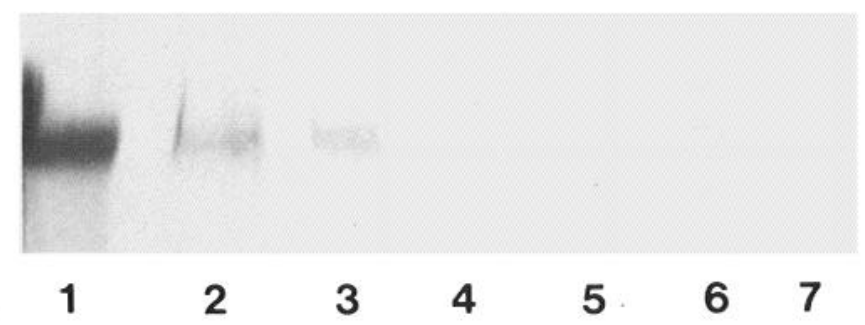

V C1.1

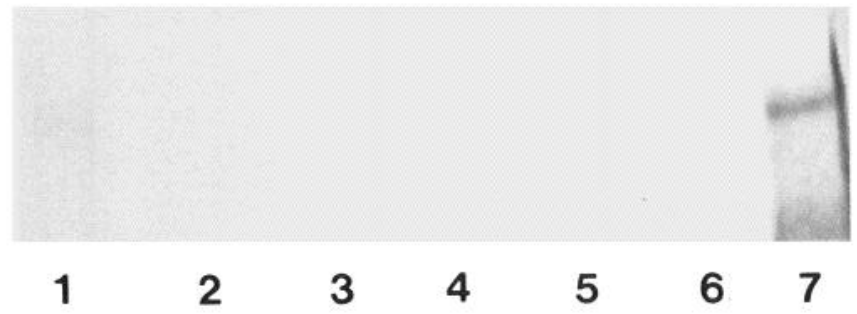

Figure 5. Resolution of Cat-301 and VC1.1 HMW antigens. Partially purified antigen (see Fig. 4) was immunoadsorbed with 6 sequential rounds of Cat-301-coated beads (lanes 1-6). The unbound fraction in each case was applied to a fresh aliquot of antibody beads. After immunodepletion with Cat-301, the unbound fraction was applied to VC1.1coated beads (lane 7). All immunoprecipitates were collected, washed, and applied to duplicate gels for blotting with Cat-301 (top) or VCl.1 (bottom). Note that the final immunoprecipitate stained intensely for the HMW VC1.1 antigen but not at all for Cat-301.

at $95-105 \mathrm{kDa}$, previously found to be enriched in cerebral cortical membranes (Arimatsu et al., 1987), was not detected in the retina sample. Identical samples stained with Cat-301 showed that the $680-\mathrm{kDa}$ proteoglycan was present in the cerebral cortex but not the retina. As we report below, the presence of some VC1.1 antigens, but not Cat-301 antigens, in the retina is supported by immunocytochemical staining.

\section{Cat-301 and VC1.1 recognize overlapping sets of antigens}

The Cat-301 antigen can be partially purified by a combination of DEAE chromatography in $8 \mathrm{M}$ urea at $\mathrm{pH} 4.9$ and gel filtration on Sepharose CL-2B (Zaremba et al., 1989). We determined that this Sepharose fraction also contained the HMW VC1.1 antigen, thus demonstrating its partial copurification with the Cat-301 antigen. This observation, combined with the similarity in the electrophoretic mobility of Cat-301 and VC1.1 antigens on Western blots, raised the possibility that the 2 antibodies might recognize different epitopes on the same molecule. To test this possibility directly, we immunoprecipitated the Sepharose-purified fraction with each antibody, and then analyzed the adsorbed species by Western blot analysis using both antibodies.

As shown in Figure 4, Cat-301 immunoprecipitated antigens contained VC1.1 immunoreactivity, and VC1.1 immunoprecipitated antigens contained Cat-301 immunoreactivity. In each case, the relative intensity of staining with the 2 antibodies was dependent on the precipitating antibody. In the Cat-301 immunoprecipitate, Cat-301 staining was far more intense than 
VC1.1 staining. In the VC1.1 immunoprecipitate, the reverse was true. These observations are not consistent with the existence of a single class of HMW molecules reacting with both antibodies. Rather, they suggested the presence of several distinct molecules, only some of which carry both the Cat-301 and VC1.1 epitopes.

This hypothesis was confirmed in a series of immunodepletion experiments (Figs. 5, 6). In these studies, an aliquot of Sepharose-purified antigen was repeatedly immunoprecipitated with one antibody, and the remaining soluble material was then tested for the presence of antigens recognized by the second antibody. In the first experiment, several consecutive rounds of immunoprecipitation with Cat-301 produced precipitates with decreasing immunostaining for both Cat-301 and VC1.1 (Fig. 5 , lanes $1-6$, top, bottom). Subsequent immunoprecipitation with $\mathrm{VCl} 1.1$ produced an HMW species that reacted with $\mathrm{VC1} 1.1$, but not with Cat-301 (lane 7, bottom, top). This indicated the existence of separate VC1.1 antigens that were not recognized by Cat-301.

Figure 6 shows a second series of immunodepletions, this time performed first with VC1.1. These experiments indicated that some HMW molecules possessed both Cat-301 and VC1.1 epitopes, while other HMW molecules contained one or the other epitope alone. After several consecutive rounds of immunoprecipitation with $\mathrm{VCl} 1$, the final precipitates showed no detectable VC1.1 staining (Fig. 6, lanes 1-5, top) and were markedly reduced in Cat-301 staining (lanes 1-5, bottom). Immunoprecipitation of the remaining depleted supernatant with Cat301 produced a precipitate that stained intensely with Cat-301, but not at all with VC1.1 (lane 6 , bottom, top). The results of the immunodepletion experiments (Figs. 5, 6) are consistent with the existence of 3 classes of HMW antigens: Cat $-301^{+/}$ $\mathrm{VCl} \cdot 1^{+}$, Cat-301+/VC1.1 ${ }^{-}$, and Cat-301-/VC1.1 ${ }^{+}$.

\section{The HMW VCl.1 antigen is a glycoconjugate}

Treatment of cortical membranes with $N$-glycanase has been shown to abolish VC1.1 immunoreactivity on Western blots, indicating that the VC1.1 epitope is probably an $\mathrm{N}$-linked carbohydrate (Barnstable and Naegele, 1989). Additional studies showed that the lower molecular weight glycoproteins defined by VC1.1 also share the HNK-1 carbohydrate epitope (Barnstable and Naegele, 1989). As shown in Figure 7, the HMW antigens immunoprecipitated by $\mathrm{VC1} .1$ and Cat-301 also reacted with the anti-HNK-1 antibodies (lanes 1, 2).

The Cat-301 antigen was previously shown to be a chondroitin sulfate proteoglycan (Zaremba et al., 1989). Enzymatic and immunological tests were performed on the Sepharose-purified fraction to define further carbohydrate moieties on the HMW antigens. As shown in Figure 7, chondroitinase treatment completely shifted Cat-301 immunoreactivity to a new position (lanes $3,4)$. However, this same treatment resulted in only a partial shift in the HMW VC1.1 immunoreactive band (lanes 5, 6). Most of the VC1.1 immunoreactivity remained at the control position; the only new VCl.1 band generated by chondroitinase migrated to the precise position of the chondroitinase-generated band defined by Cat-301. This digestion suggested that not all of the HMW VC1.1 antigens are chondroitin sulfate proteoglycans. The subset of HMW VC1.1 antigens that were sensitive to chondroitinase may correspond to the set that also carried the Cat-301 epitope.

Additional experiments indicated that some of the HMW VC1.1 antigens contain keratan sulfate moieties. Monoclonal

\section{VC1.1}
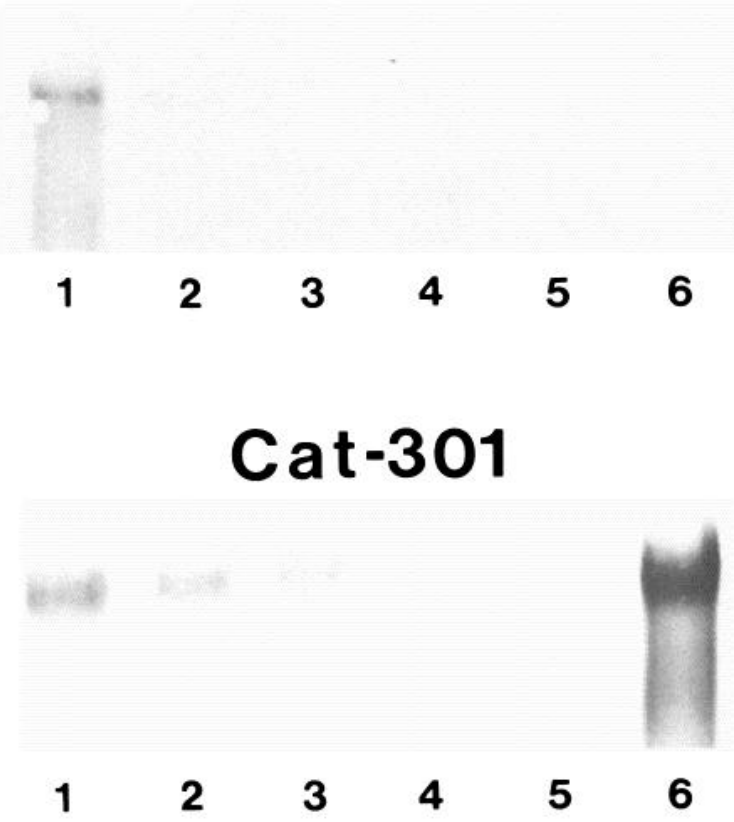

Figure 6. Presence and absence of VC1.1 epitopes on subsets of Cat301 proteoglycans. Partially purified antigen was immunoadsorbed with 5 rounds of $\mathrm{VC1} 1$-1-coated beads (lanes 1-5), then immunoadsorbed with Cat-301-coated beads (lane 6). Immunoprecipitates were applied to duplicate gels and blotted with VC1.1 (top) or Cat-301 (bottom). Some Cat-301 proteoglycans contained VC1.1 immunoreactivity (lanes 1-5; see also Figs. 4,5 , while other Cat-301 proteoglycans were $\mathrm{VCl} .1$ negative (lane 6).

antibodies to keratan sulfate chains reacted with the HMW antigen in VC1.1 immunoprecipitates, but not with the 680 $\mathrm{kDa}$ antigen in Cat-301 immunoprecipitates (Fig. 7, lanes 710). Furthermore, following incubation of samples with keratanase, the HMW VC1.1 antigen still stained with VC1.1 but no longer stained with anti-keratan sulfate antibody (lanes 11, 12). We therefore conclude that some of the HMW VC1.1 antigens (excluding those molecules that are also positive for Cat$301)$ are proteoglycans with keratan sulfate chains or are tightly associated with such proteoglycans. We have recently determined that anti-keratan sulfate antibodies also stain the surfaces of subsets of neurons in the adult cat CNS (Sam Zaremba and Susan Hockfield, unpublished observations).

\section{Double-label immunofluorescence defines three sets of Cat-301- and VC1.1-positive neurons}

Previous studies showed that both Cat-301 and VC1.1 recognize subpopulations of neurons in area 17 of the cat cerebral cortex (Hockfield et al., 1983; Hendry et al., 1988; Naegele et al., 1988; Guimarães et al., 1990; reviewed in Naegele and Barnstable, 1989). In the present study, we used double-label immunofluorescence to determine the overlap in the distribution of the Cat-301 and VC1.1 antigens in different CNS regions. The anatomical findings were consistent with the biochemical studies in that multiple classes of antibody-positive neurons were found: those that were positive for both antibodies and those that were positive for each antibody alone. In addition to labeling cell bodies, both antibodies also stain elements of the neuropil. We 
Lyase

$\begin{array}{ccc}\text { Precipitating } & \text { VC } & \text { Cat } \\ \text { Antibody } & 1.1 & 301\end{array}$
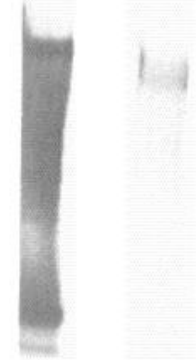

Staining Antibody

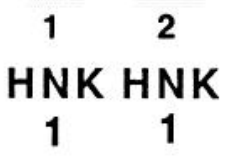

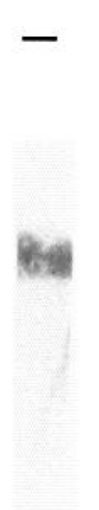

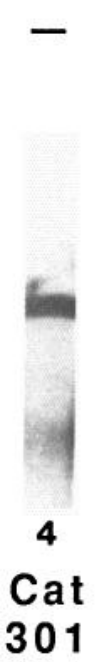

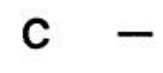
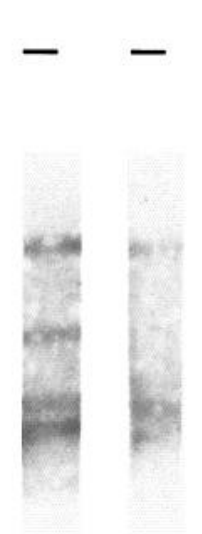

$\begin{array}{cc}5 & 6 \\ V C & V C \\ 1.1 & 1.1\end{array}$

\section{VC VC}

1.11 .1

Cat

301301

VC

1.1

VC
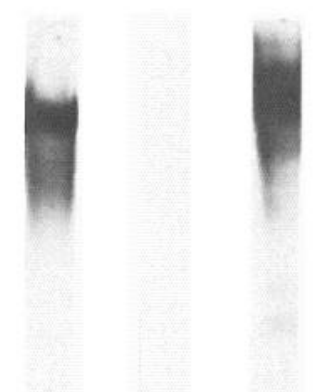

$\begin{array}{cccccc}7 & 8 & 9 & 10 & 11 & 12 \\ \text { VC } & \text { KS } & \text { Cat } & \text { KS } & \text { VC } & \text { KS } \\ 1.1 & & 301 & & 1.1 & \end{array}$

Figure 7. Carbohydrate moieties on VC1.1 and Cat-301 HMW antigens. Lanes 1, 2, Partially purified antigen was immunoadsorbed either with VC1.1-coated (lane 1) or Cat-301-coated (lane 2) beads. Immunoprecipitates were electrophoresed, transferred, and stained with HNK-1 antibody. Both immunoprecipitates contained HMW antigens that reacted with anti-HNK-1. Lanes 3-6, Antigen was incubated in the absence (lanes 3,6$)$ or presence (lanes 4,5$)$ of chondroitinase $\mathrm{ABC}(C)$ and was stained with the indicated antibodies. Chondroitinase completely shifted the Cat-301 immunostaining to a lower apparent molecular weight, but much of the HMW VC1.1 immunostaining remained at the control position. The only new VC1.1 immunoreactive band electrophoresed to the position of the chondroitinase-treated Cat-301 antigen (cf. lanes 5, 4). Lanes $7-12$, Antigen was incubated in the absence (lanes $7-10)$ or presence (lanes 11,12$)$ of keratanase $(K)$, then immunoadsorbed, electrophoresed, and stained with the indicated antibodies. Some or all of the HMW VC1.1 antigens possessed keratan sulfate moieties, but the Cat-301 antigen did not. In controls, keratanase treatment had no effect on VC1.1 staining, but abolished staining with anti-keratan sulfate.

have limited our analysis of colocalization to cell bodies. Although the immunoreactive elements in the neuropil have not yet been identified in all regions, it seems likely that in some areas, most of this staining is due to the presence of immunoreactivity on dendrites of cells whose perikarya are also clearly outlined.

Retina. In agreement with previously reported studies, $\mathrm{VC1} .1$ stained the inner and outer plexiform layers and a subset of horizontal cells and amacrine cells within the inner nuclear layer (Fig. 8A). Cat-301 immunoreactivity was not detected in the retina (Fig. $8 B$ ).

Dorsal lateral geniculate nucleus. VC1.1 immunoreactivity was not associated with neuronal cell bodies in the LGN (Fig. $8 C$; see also Arimatsu et al., 1987). In contrast, Cat-301 strongly labeled large neurons within the cat LGN (Fig. 8D; Hockfield et al., 1983; Hendry et al., 1984). Previous studies showed that the Cat-301+ neurons correspond to the class I or Y-cells of the LGN (Sur et al., 1988; Hockfield and Sur, 1990). Both VC1.1 and Cat-301 also produced a light, diffuse staining of the neuropil within the LGN. In contrast to the differences observed in the LGN, VC1.1 and Cat-301 immunoreactivity showed complete overlap on individual neurons in 2 other thalamic nuclei, the perigeniculate nucleus (not shown), and thalamic reticular nucleus (Fig. 9, $A, B$ ).

Area 17. VC1.1 immunoreactivity outlined a subset of nonpyramidal neurons primarily in cortical layer 4 and some additional nonpyramidal neurons in other layers (Fig. $8 E$ ), confirming previous observations (Arimatsu et al., 1987; Naegele et al., 1988). In contrast, Cat-301 immunoreactivity outlined neurons with both pyramidal and nonpyramidal morphologies in layers 2-5 (Fig. 8F), as reported previously (Hockfield et al., 1983; Hendry et al., 1988; Guimarães et al., 1990). Layers 1 and 6 contained very few neurons positive for either antibody. A substantial number of neurons in the middle cortical layers, mostly in layer 4, were immunoreactive for both Cat-301 and VC1.1 (Fig. 8, $E, F$ ). The overlap in the distribution of Cat-301 and VC1.1 immunoreactivity was determined by counting immunoreactive neurons in layers $2-6$ of area 17 . These counts revealed that $55 \%$ of the immunoreactive neurons were $\mathrm{VCl} .1^{+}$ Cat $-301^{+}(336 / 614), 44 \%$ were VC1.1-/Cat-301+ $(273 / 614)$ and only $1 \%$ were $\mathrm{VCl} 11^{+} / \mathrm{Cat}-301^{-}(5 / 614)$.

Spinal cord. Much of the VC1.1 immunoreactivity in the spinal cord was associated with axonal surfaces in the white matter; however, some labeling of neuronal cell bodies was also evident (Fig. 9E). In contrast, Cat-301 staining was largely limited to the grey matter of the spinal cord and outlined many different classes of neurons, including motor neurons (Fig. 9F). Many of the Cat-301-positive neurons also exhibited weak VC1.1 immunoreactivity along the cell surfaces.

Cerebellum. VC1.1 and Cat-301 both outlined subsets of neurons in the deep nuclei of the cerebellum. In this region, there was complete overlap in the immunoreactive populations (Fig. $9, C, D)$. However, a very different distribution was found for the 2 markers in the cerebellar cortex. In this region, $\mathrm{VCl}$.1 immunoreactivity was present along the surfaces of distal dendrites in the molecular layer and also stained some elements that extended across the Purkinje cell bodies (Fig. 10, $B, C$, arrowheads). It is not yet clear which cell type gives rise to these processes. As reported previously, Cat-301 stained only a relatively rare cell type, the Lugaro cell, whose cell bodies are 


\section{VC1.1}
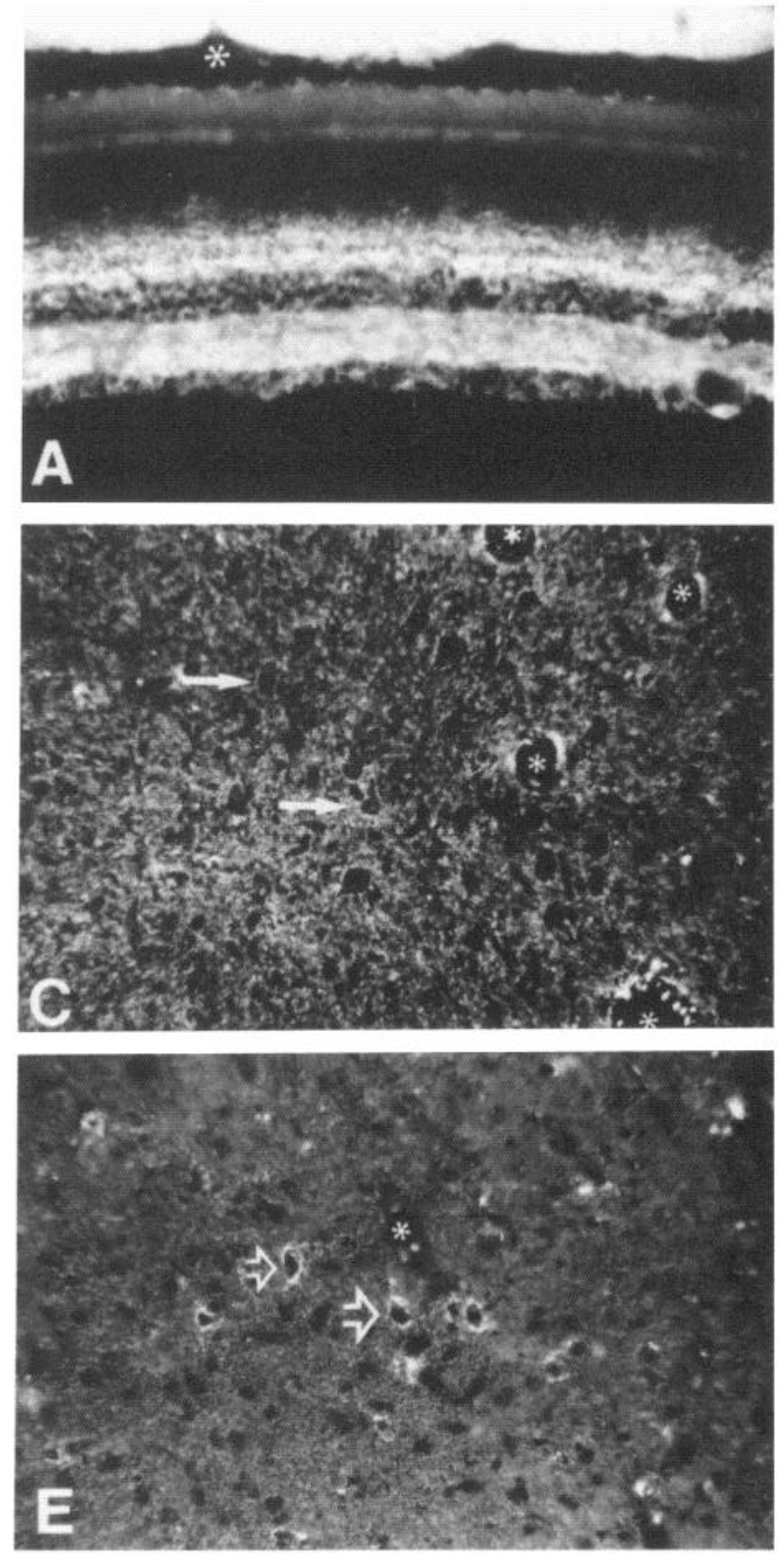

\section{CA T - 301}
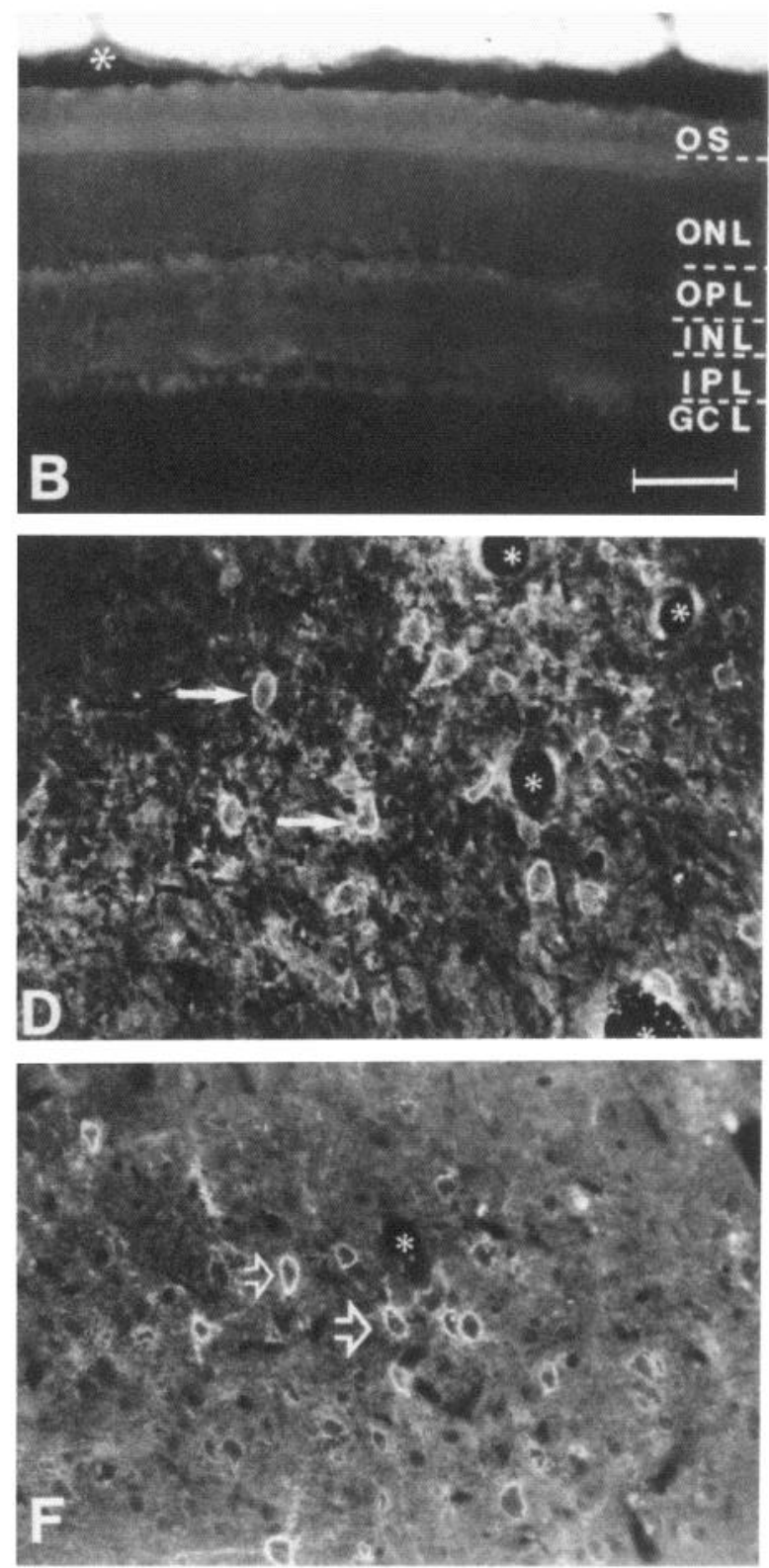

Figure 8. Comparison of VC1.1 and Cat-301 immunoreactivity in the visual system using double-label immunofluorescence. In the retina, VC1.1 stained the inner and outer plexiform layers $(I P L, O P L)$ and scattered subsets of neurons in the inner nuclear layer $(I N L)(A)$. Cat-301 immunoreactivity was not present in the retina $(B)$. In the dorsal LGN, VCl.1 immunoreactivity was associated with elements in the neuropil, giving a fine granular appearance to the staining $(C)$. Cat-301 immunoreactivity in the LGN was primarily associated with large neurons that were negative or faintly VCl.1 positive ( $C, D$; solid arrows). In the visual cortex, VC1.1 and Cat-301 immunoreactivity colocalized on some but not all neurons $(E, F ;$ open arrows). Asterisks indicate blood vessels to facilitate matching sets of photomicrographs. $O S$, outer segments; $O N L$, outer nuclear layer; $G C L$, ganglion cell layer. Scale bar, $50 \mu \mathrm{m}$.

located just below the Purkinje layer (Fig. 10A; Hockfield et al., 1983; Sahin and Hockfield, 1990).

\section{Discussion}

The present study provides support for the hypothesis that distinct members of a family of HMW, surface-associated glycoconjugates are expressed selectively by subsets of neurons. Monoclonal antibodies Cat-301 and VC1.1 recognize surfaceassociated antigens on 3 classes of immunoreactive neurons in the cat CNS. Although the 2 antibodies react with different neuronal subsets in some brain regions, both antibodies react with the same neuronal subset in other regions. Our biochemical studies now indicate that these antibodies recognize distinct HMW, acidic antigens that contain sulfated carbohydrate chains characteristic for proteoglycans.

Diversity among proteoglycans in the developing and the adult mammalian brain has recently become evident, caused in part by the introduction of new antibody probes and other techniques. Margolis and colleagues (Gowda et al., 1989) have dem- 


\section{VC1.1}
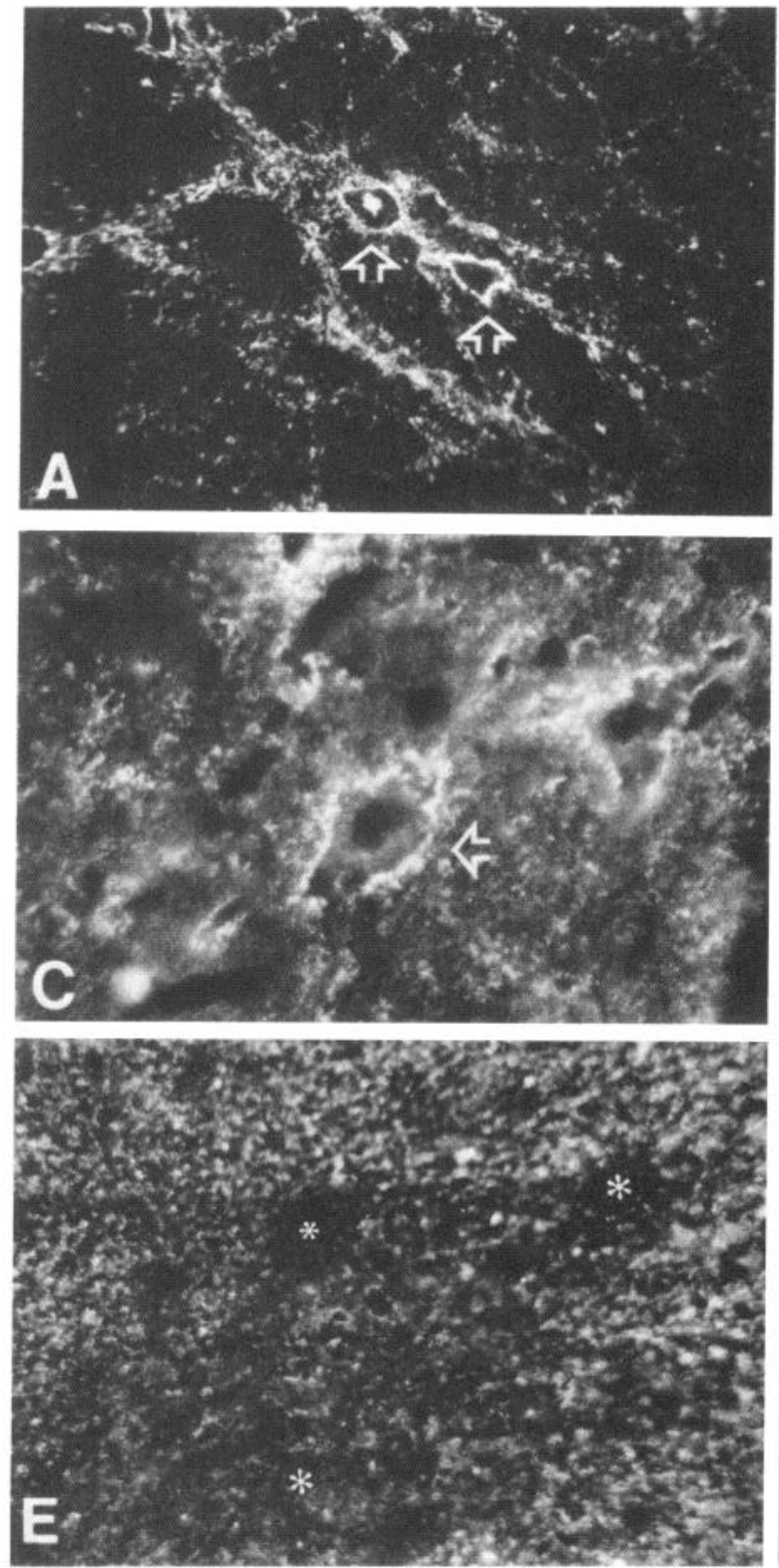

\section{CAT - 301}
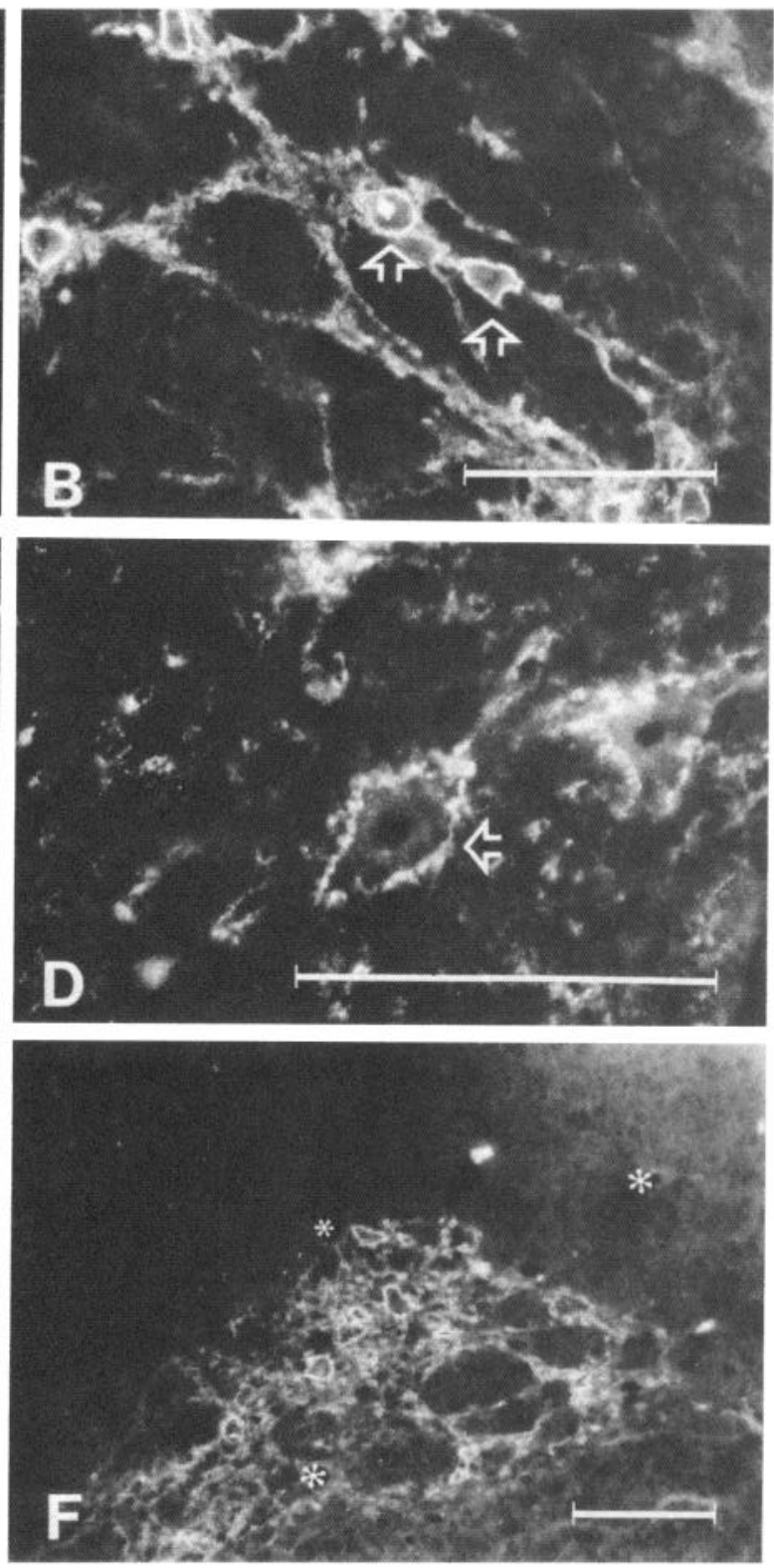

Figure 9. Comparison of VC1.1 and Cat-301 immunoreactivity in some thalamic and cerebellar nuclei and spinal cord. In the thalamic nucleus reticularis, both neurons and processes were strongly VC1.1 and Cat-301 immunoreactive $(A, B)$. In the deep cerebellar nuclei, VC1.1 and Cat301 immunoreactivity labeled the same population of neurons $(C, D)$. In the spinal cord, VC1.1 immunoreactivity predominated in the fiber tracts and, to a lesser extent, in the gray matter $(E)$. In contrast, Cat-301 immunoreactivity was associated exclusively with neurons and neuronal processes in the gray matter $(F)$. Asterisks indicate blood vessels to facilitate matching sets of photomicrographs. Scale bars, $100 \mu \mathrm{m}$.

onstrated that chondroitin sulfate chains are present on a number of different polypeptide cores in the rat brain. The occurrence of these cores, ranging in size from 80 to $400 \mathrm{kDa}$, is likely to reflect distinct transcription products, because comparable diversity is obtained by in vitro translation from brain mRNA followed by immunoprecipitation with an antiserum to chondroitin sulfate proteoglycans. Further evidence for diversity is provided by the work of Oohira et al. (1988), in which a rat brain proteoglycan preparation was treated with chondroitinase to generate several putative core polypeptides that differ from one another by tryptic peptide maps. In addition, proteoglycan diversity is evident in the finding that brain tissue contains heparan sulfate proteoglycans (Klinger et al., 1985; Ripelino and Margolis, 1989), as well as keratan sulfate moieties (Weinstein et al., 1983; Eronen et al., 1985; Krusius et al., 1986; Funderburgh et al., 1987). Immunohistochemical studies indicate that several brain proteoglycans are localized on the surfaces of neurons (Hockfield and McKay, 1983; Aquino et al., 1984; Matthew et al., 1985; Hoffman et al., 1988; Watanabe et al., 1989; Zaremba et al., 1989; Fujita et al., 1989). One such 

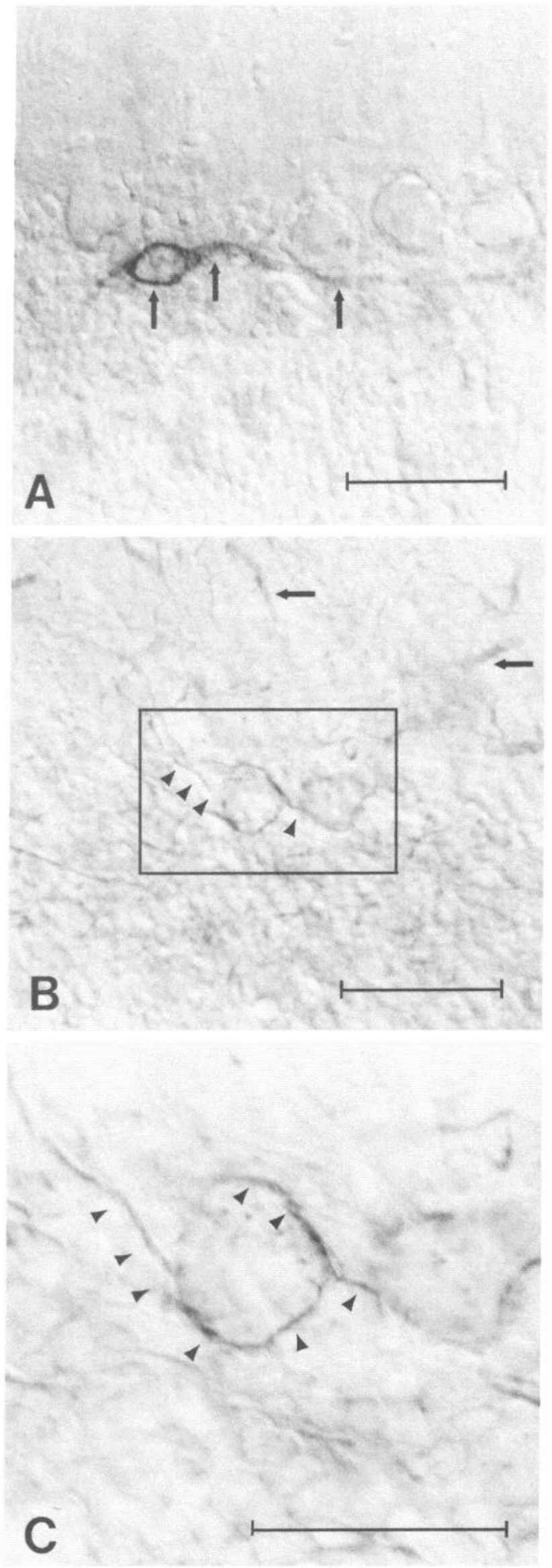

molecule, cytotactin-binding proteoglycan, has been proposed to function in cell-cell adhesion by serving as a ligand for cytotactin (Chuong et al., 1987; Hoffman and Edelman, 1987; Hoffman et al., 1988). The demonstrated roles of proteoglycans in adhesion and neurite outgrowth, combined with their molecular and anatomical diversity, has led to the suggestion that individual members of a family of proteoglycans may be present on the surfaces of distinct subpopulations of neurons and might be involved in neural morphogenesis.

Four lines of evidence indicate that Cat-301 and VC1.1 epitopes define multiple HMW glycoconjugates. First, the HMW $\mathrm{VC} 1.1$ species is present in Western blots of retina homogenates, where there is no detectable Cat-301 antigen. This finding is consistent with localization studies showing VC1.1 but not Cat301 immunoreactivity in the retina. Second, in samples that do contain both antigens (e.g., cortex), treatment with chondroitinase only partially digests the HMW VC1.1 band, while the Cat301 band is completely shifted to a new position. Third, the HMW antigens isolated by VC1.1 immunoprecipitation react with anti-keratan sulfate antibodies, while the antigen isolated by Cat-301 immunoprecipitation does not. Finally, immunoadsorption and depletion experiments show that a substantial amount of the HMW VC1.1 antigen remains after removal of all or most of the Cat-301 antigen. Similarly, substantial amounts of Cat $-301^{+} / \mathrm{VCl} .1^{-}$antigen are detected after immunodepletion with VC1.1. The Cat $-301^{+} / \mathrm{VCl} 11^{+}$and $\mathrm{VCl} .1^{+} / \mathrm{Cat}-301^{-}$molecules identified by immunodepletion may correspond to the chondroitinase-sensitive and -insensitive HMW VC1.1 bands, respectively.

We have therefore identified 3 sets of HMW antigens: Cat$301^{+} / \mathrm{VCl} .1^{-}$, Cat-301-/VC1.1 ${ }^{+}$, and Cat $-301^{+} / \mathrm{VCl} \cdot 1^{+}$. These sets may not be absolutely distinct, because an antigen that appears negative for one antibody may in fact possess the relevant epitope, but in amounts below the limit of detection of the methods employed here. It is clear, however, that the antigen sets as defined here are different from one another in their relative Cat-301:VC1.1 staining ratio.

The presence of multiple, biochemically defined HMW antigens is consistent with the distribution of immunoreactive cell bodies in different CNS regions. (It should be emphasized that, in some brain regions, staining with $\mathrm{VCl} 1.1 \mathrm{might}$ be due to some of the other VC1.1 antigens.) Both antibodies label identical sets of neurons in the deep cerebellar nuclei and in some thalamic nuclei. In other regions, such as the cerebellar cortex and LGN, the antibodies recognize different cell groups. In the LGN, Cat-301-labeled neurons were previously shown to be class I, or Y-cells (Sur et al., 1988; Hockfield and Sur, 1990); these neurons are not labeled by $\mathrm{VC1}$.1. In contrast, in the retina, VC1.1 labels some horizontal and amacrine cell types, but Cat301 does not label any cellular elements. In the primary visual cortex, a partial overlap in labeling is observed. VCl.1 stains a subset of area 17 neurons primarily in layer 4 , which were pre-

Figure 10. Comparison of VC1.1 and Cat-301 immunoreactivity in cerebellar cortex. Cat-301 immunoperoxidase staining was observed along the cell bodies and dendrites of Lugaro cells ( $A$, arrows). VC1.1 immunoreactivity was present on dendrites in the molecular layer $(B$, arrows) and on fibers present in all layers (arrowheads). The boxed area in $B$ is shown at a higher magnification in $C$, where the immunoreactive fibers cross a Purkinje cell body and give rise to small branchlets before entering the molecular layer. Scale bars, $20 \mu \mathrm{m}$. 
viously shown to be GABAergic interneurons (Naegele et al., 1988; Naegele and Barnstable, 1989). Cat-301 labeling in area 17 is more extensive and includes both GABAergic interneurons and projection neurons (Hendry et al., 1988; Guimarães et al., 1990).

Area 17 of the cat cerebral cortex contains a number of cells that are Cat-301 positive and $\mathrm{VCl} 1.1$ negative, consistent with the biochemical identification of a Cat-301+/VC1.1- molecule. A far smaller number of cells are VC1.1 positive and Cat-301 negative. However, biochemical analysis of homogenates from the total cortex suggests the presence of a substantial population

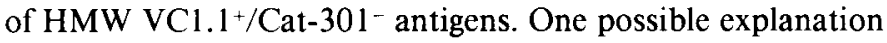
for this apparent discrepancy is that $\mathrm{VCl} .1^{+/}$Cat $-301^{-}$neurons may be more abundant in areas of the cortex outside area 17 .

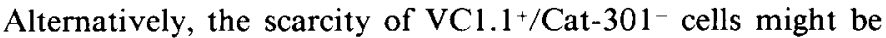
due to the difficulty in resolving antigenically related molecules at the single-cell level. An individual neuron that simultaneously carries both $\mathrm{Cat}-301^{+} / \mathrm{VC1} .1^{-}$and $\mathrm{VC1} .1^{+} / \mathrm{Cat}-301^{-}$antigens might be indistinguishable from a neuron that carries the Cat$301^{+} / \mathrm{VC} 1.1^{+}$antigen. A third possibility is that some $\mathrm{VCl} .1^{+}$ Cat $-301^{-}$molecules may be located primarily on elements in the neuropil, rather than on cell bodies.

In the case of Cat-301, previous studies have provided strong evidence that the biochemically defined chondroitin sulfate proteoglycan is the surface-associated antigen observed histologically. Monoclonal antibody Cat-304, which produces staining patterns identical to those seen with Cat-301 (Guimarães et al., 1990), cross-reacts with the Cat-301 proteoglycan, but at a distinct epitope (Zaremba et al., 1989). These findings indicate that the molecules defined biochemically and histologically are identical. In addition, immunoprecipitation of radioiodinated cortical membranes with Cat-301 identifies only the $680-\mathrm{kDa}$ antigen (S. Zaremba, unpublished results). At present, it is difficult to determine which of the antigens recognized by VC1.1 are responsible for cell-surface staining. Western blot analysis reveals 4 major immunoreactive bands with relative molecular masses of 95-105 kDa, $145 \mathrm{kDa}, 170 \mathrm{kDa}$, and $650-700 \mathrm{kDa}$. Because the $95-105-\mathrm{kDa}$ band is not detected in Western blots of retinal homogenates, the surface staining in the retina is probably due to a combination of the other hands.

The antigens identified by Cat-301 and VC1.1 illustrate several possible mechanisms by which molecular diversity of surface molecules can be achieved. Because the Cat-301 epitope has been shown to be on a polypeptide portion of the proteoglycan molecule, this epitope is probably regulated at the transcriptional or translational level. In contrast, because the VC1.1 epitope is a carbohydrate, this epitope is probably regulated at the posttranslational level by changes in the activities of specific glycosyltransferases or glycosylhydrolases.

These mechanisms may be used by individual neurons to generate a diversity of cell-surface glycoconjugates, each with distinct functional roles. Recent work has demonstrated that the VCl.l epitope is present on members of a family of neuronal adhesion molecules, including myelin-associated glycoprotein and the neural cell adhesion molecule, N-CAM (Barnstable and Naegele, 1989). The HMW VC1.1 glycoconjugate described in the present report may also belong to this family of adhesion molecules. If so, then regulation of VCl.1 epitope expression might regulate levels of adhesivity on a specific cell-surface molecule.

The Cat-301 antigen is not likely to play a role in adhesion events that take place early in development, because its expres- sion is delayed until the period of synaptic plasticity is drawing to a close (Hockfield et al., 1983; Kalb and Hockfield, 1988; Sur et al., 1988). This delayed expression, along with the regulation of antigen expression by neuronal activity during early postnatal development, has led to the suggestion that the Cat-301 proteoglycan might play a role in the stabilization of the mature synaptic structure (Kalb and Hockfield, 1988; Sur et al., 1988; Hockfield et al., 1989; Guimarães et al., 1990).

The existence of HMW molecules that are Cat-301 negative but $\mathrm{VCl} .1$ positive raises the possibility that multiple HMW cell-surface glycoconjugates are involved in maintaining the mature structure of the nervous system. The HMW antigens described in this paper are restricted to relatively few neuronal cell types in the adult nervous system. As new probes for other HMW cell-surface molecules become available, it will be interesting to determine whether they are expressed on different sets of neurons or whether only a distinct class of neurons express these molecules.

\section{References}

Abo T, Balch CM (1981) A differentiation antigen of human NK and $\mathrm{K}$ cells identified by a monoclonal antibody (HNK-1). J Immunol 127:1024-1029.

Aquino DA, Margolis RU, Margolis RK (1984) Immunocytochemical localization of a chondroitin sulfate proteoglycan in nervous tissue II. Studies in developing brain. J Cell Biol 99:1130-1139.

Arimatsu Y, Naegele JR, Barnstable CJ (1987) Molecular markers of neuronal subpopulations in layers 4,5 and 6 of cat primary visual cortex. J Neurosci 7:1250-1263.

Barnstable CJ, Naegele JR (1989) Biochemical analysis of cortical cell surface molecules recognized by antibodies VC1.1 and VC5.1. Soc Neurosci Abstr 15:892.

Bensadoun A, Weinstein D (1976) Assay of protein in the presence of interfering materials. Anal Biochem 70:241-250.

Caterson B, Christner JE, Baker JR (1983) Identification of a monoclonal antibody that specifically recognizes corneal and skeletal keratan sulfate. J Biol Chem 258:8848-8854.

Chou KH, Ilyas AA, Evans JE, Quarles RH, Jungalwala FB (1985) Structure of a glycolipid reacting with monoclonal IgM in neuropathy and with HNK-1. Biochem Biophys Res Commun 128:383-388.

Chuong C-M, Crossin KL, Edelman GM (1987) Sequential expression and differential functions of multiple adhesion molecules during the formation of cerebellar cortical layers. J Cell Biol 104:331-342.

Eldred WD, Zucker C, Karten HJ, Yazulla S (1983) Comparison of fixation and penetration enhancement techniques for use in ultrastructural immunocytochemistry. J Histochem Cytochem 31:285292.

Eronen I, Mononen T, Mononen I (1985) Isolation and partial characterization of keratan sulfate from human brain. Biochim Biophys Acta 843:155-158.

Fujita SC, Tada Y, Murakami F, Hayashi M, Matsumura M (1989) Glycosaminoglycan-related epitopes surrounding different subsets of mammalian central neurons. Neurosci Res 7:117-130.

Funderburgh JL, Caterson B, Conrad GW (1987) Distribution of proteoglycans antigenically related to corneal keratan sulfate proteoglycan. J Biol Chem 262:11634-11640.

Gowda DC, Margolis RU, Margolis RK (1989) Presence of the HNK-1 epitope on $\operatorname{poly}(N$-acetyllactosaminyl) oligosaccharides and identifcation of multiple core protein in the chondroitin sulfate proteoglycans of brain. Biochemistry 28:4468-4474.

Guimarães A, Zaremba S, Hockfield S (1990) Molecular and morphological changes in the cat lateral geniculate nucleus and visual cortex induced by visual deprivation are revealed by monoclonal antibodies Cat-304 and Cat-301. J Neurosci 10:3014-3024.

Hendry SHC, Hockfield S, Jones EG, McKay R (1984) Monoclonal antibody that identifies subsets of neurones in the central visual system of monkey and cat. Nature 307:267-269.

Hendry SHC, Jones EG, Hockfield S, McKay RDG (1988) Neuronal populations stained with the monoclonal antibody Cat-301 in the mammalian cerebral cortex and thalamus. J Neurosci 8:518-542.

Hockfield S (1987) A novel cerebellar neuron identified by a mono- 
clonal antibody generated with immunosuppressive and rapid immunization strategies. Science 237:67-70.

Hockfield S, McKay RDG (1983) A surface antigen expressed by a subset of neurons in the vertebrate central nervous system. Proc Nat Acad Sci USA 80:5758-5761.

Hockfield S, Sur M (1990) Monoclonal antibody Cat-301 identifies Y-cells in the dorsal lateral geniculate nucleus of the cat. J Comp Neurol (in press).

Hockfield S, McKay RD, Hendry SHC, Jones EG (1983) A surface antigen that identifies ocular dominance columns in the visual cortex and laminar features of the lateral geniculate nucleus. Cold Spring Harbor Symp Quant Biol 48:877-889.

Hockfield S, Kalb R, Guimarães A (1989) Experience-dependent expression of neuronal cell-surface molecules. In Neuroimmune Networks: Physiology and Disease (Goetzl E, Liss AR, eds), pp 57-63.

Hoffman S, Edelman GM (1987) A proteoglycan with HNK-1 antigenic determinants is a neuron-associated ligand for cytotactin. Proc Nat Acad Sci USA 84:2523-2527.

Hoffman S, Crossin KL, Edelman GM (1988) Molecular forms, binding functions and developmental expression patterns of cytotactin and cytotactin-binding proteoglycan, an interactive pair of extracellular matrix molecules. J Cell Biol 106:519-532.

Kalb RG, Hockfield S (1988) Molecular evidence for early activity. dependent development of hamster motor ncurons. J Ncurosci 8: 2350-2360.

Klinger MM, Margolis RU, Margolis RK (1985) Isolation and characterization of the heparan sulfate proteoglycans of brain: use of affinity chromatography on lipoprotein lipase-agarose. J Biol Chem 260:4082-4090.

Kohler G, Milstein C (1975) Continuous cultures of fused cells secreting antibody of predefined specificity. Nature 256:495-497.

Kruse J, Mailhammer R, Wernecke H, Faissner A, Sommer I, Goridis C, Schachner M (1984) Neural cell adhesion molecules and myelinassociated glycoprotein share a common carbohydrate moiety recognized by monoclonal antibodies L2 and HNK-1. Nature 311:153155.

Krusius T, Finne J, Margolis RK, Margolis RU (1986) Identification of an O-glycosidic mannose-linked sialylated tetrasaccharide and keratan sulfate oligosaccharides in the chondroitin sulfate proteoglycan of brain. J Biol Chem 261:8237-8242.

Laemmli UV (1970) Cleavage of structural protein during assembly of the head of bacteriophage T4. Nature 227:680-685.

Margolis RU, Margolis RK (1989) Nervous tissue proteoglycans. Dev Neurosci 11:276-288.

Matthew WD, Greenspan RJ, Lander AD, Reichardt LF (1985) Immunopurification and characterization of a neuronal heparan sulfate proteoglycan. J Neurosci 5:1842-1850.
McKay RDG, Hockfield S (1982) Monoclonal antibodies distinguish antigenically discrete neuronal types in the vertebrate central nervous system. Proc Nat Acad Sci USA 79:6747-6751.

Mehmet H, Scudder P, Tang PW, Hounsell EF, Caterson B, Feizi T (1986) The antigenic determinants recognized by three monoclonal antibodies to keratan sulphate involve sulphated hepta- or larger oligosaccharides of the poly( $N$-acetyllactosamine) series. Eur J Biochem 157:385-391.

Naegele JR, Barnstable CJ (1989) Molecular determinants of GABAergic local circuit ncurons in the visual cortex. Trends Neurosei 12:28-34.

Naegele JR, Arimatsu Y, Schwartz P, Barnstable CJ (1988) Selective staining of a subset of GABAergic neurons in cat visual cortex by monoclonal antibody VC1.1. J Neurosci 8:79-89.

Oohira A, Matsui F, Matsuda M, Takida Y, Kuboki Y (1988) Occurrence of three distinct molecular species of chondroitin sulfate proteoglycan in the developing rat brain. J Biol Chem 263:1024010246.

Ripelino JA, Margolis RU (1989) Structural properties of the heparan sulfate proteoglycans of brain. J Neurochem 52:807-812.

Ruoslahti E (1988) Structure and biology of proteoglycans. Ann Rev Cell Biol 4:229-255.

Sahin M, Hockfield S (1990) Molecular identification of the Lugaro ccll in the cat ccrcbcllar cortcx. J Comp Neurol (in press).

Sur M, Frost DO, Hockfield S (1988) Expression of a cell surface antigen on Y-cells in the cat lateral geniculate nucleus is regulated by visual experience. J Neurosci 8:874-882.

Tang PW, Scudder P, Mehmet H, Hounsell EF, Feizi T (1986) Sulphate groups are involved in the antigenicity of keratan sulphate and mask $i$ antigen expression on their poly- $N$-acetyllactosamine backbones: an immunochemical and chromatographic study of keratan sulphate oligosaccharides after desulphation or nitrosation. Eur J Biochem 160 537-545.

Towbin H, Staehlin T, Gordon J (1979) Electrophoretic transfer of proteins from polyacrylamide gels to nitrocellulose sheets: procedure and some applications. Proc Nat Acad Sci USA 76:4350-4354.

Watanabe E, Fujita SC, Murakami F, Hayashi M, Matsumura M (1989) A monoclonal antibody identifics a novel cpitope surrounding a subpopulation of the mamalian central neurons. Neuroscience 29:645657.

Weinstein HG, Blacik LJ, Breen M, Knepper PA (1983) The presence of a keratan sulfate-like glycoconjugate in microsomes of the cerebral cortex. Biochem Biophys Res Commun 111:28-35.

Zaremba S, Guimarães A, Kalb RG, Hockfield S (1989) Characterization of an activity dependent neuronal surface proteoglycan identified with monoclonal antibody Cat-301. Neuron 2:1207-1219. 